\title{
Dynamic response of an optomechanical system to a stationary random excitation in the time domain
}

\author{
Jeremy A. Palmer ${ }^{\mathrm{a}, *}$ and Thomas L. Paez ${ }^{\mathrm{b}}$ \\ a Sandia National Laboratories, P.O. Box 5800, MS 01304, Albuquerque, NM, USA \\ ${ }^{\mathrm{b}}$ Thomas Paez Consulting, 175 Iron King, Durango, CO, USA
}

Received 13 September 2009

Revised 11 March 2010

\begin{abstract}
Modern electro-optical instruments are typically designed with assemblies of optomechanical members that support optics such that alignment is maintained in service environments that include random vibration loads. This paper presents a nonlinear numerical analysis that calculates statistics for the peak lateral response of optics in an optomechanical sub-assembly subject to random excitation of the housing. The work is unique in that the prior art does not address peak response probability distribution for stationary random vibration in the time domain for a common lens-retainer-housing system with Coulomb damping. Analytical results are validated by using displacement response data from random vibration testing of representative prototype sub-assemblies. A comparison of predictions to experimental results yields reasonable agreement. The Type I Asymptotic form provides the cumulative distribution function for peak response probabilities. Probabilities are calculated for actual lens centration tolerances. The probability that peak response will not exceed the centration tolerance is greater than $80 \%$ for prototype configurations where the tolerance is high (on the order of 30 micrometers). Conversely, the probability is low for those where the tolerance is less than 20 micrometers. The analysis suggests a design paradigm based on the influence of lateral stiffness on the magnitude of the response.
\end{abstract}

Keywords: Coulomb damping, lens, optomechanical, random vibration, retainer

\section{Introduction}

Modern electro-optical instruments for use in non-stationary platforms rely upon accurately-aligned optical elements such as lenses and mirrors to achieve target image quality. For reliable performance in the field, engineers must create optomechanical members that house and support optics such that alignment is maintained in service environments that include thermal, static inertial, shock, and random vibration loads. To this end, a predictive capability is sought that leads to a detailed understanding of how the random dynamic response of geometric optics (in this case, lenses) is impacted by parametric design changes in the optomechanical systems used to constrain them. This paper presents an engineering study on this topic. Background and development of the model and experimental validation is described in the following sections.

\subsection{Optomechanical design}

Complex instruments may include multiple installations of optomechanical systems. These systems may include optical elements, such as refractive lenses, mirrors, diffraction gratings, or photonic devices; and sub-assemblies

\footnotetext{
*Corresponding author. Tel.: +1 505284 8400; Fax: +1 505844 8168; E-mail: japalme@ sandia.gov.
} 


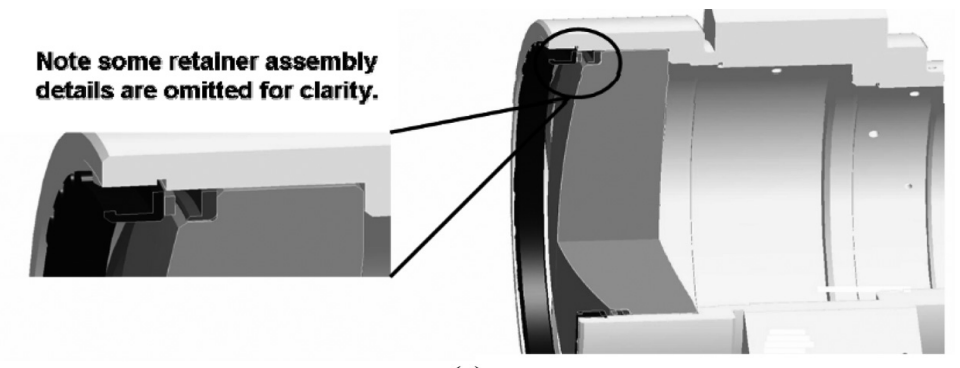

(a)

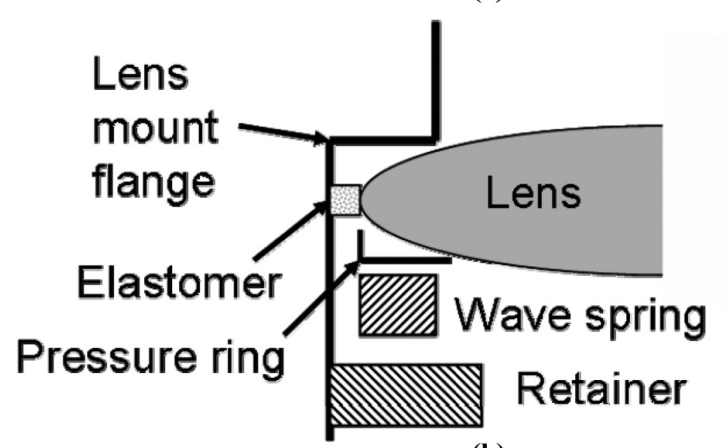

(b)

Fig. 1. (a) Single-lens optomechanical system, and (b) simplified cross-section.

of structural components that position, orient, and affix them to a datum surface or coordinate frame often with high accuracy [3,23]. A simple optomechanical system consisting of a single lens is illustrated in Fig. 1. In the configuration shown in Fig. 1 (a), a retainer with wave spring and pressure ring distributes a static normal force to the forward face of the lens, pressing it against a mounting surface in the surrounding housing. The edge of the lens is supported by discrete pads of compliant room-temperature vulcanization (RTV) elastomer (see Fig. 1 (b)) arranged symmetrically about the optical axis. RTV maintains centration of the lens without introducing damaging stress concentrations due to thermal expansion [23]. Moreover, it provides the necessary restoring force in response to dynamic inputs, discussed later. The housing, with one or more similarly-supported lenses mounts to the instrument base which transmits vibration from the environment.

\subsection{Random vibration engineering in electro-optical instruments}

Random vibration and its impact on optical alignment and image quality, particularly in aerospace applications, has been a significant consideration in engineering of electro-optical instruments. A brief review of the relevant prior art addresses methods for numerical and finite element analysis and random vibration testing with associated tools for measuring response.

Finite element models predicting the dynamic response of specific products to random excitation have been reported. Ahmad et al. describe a NASTRAN model that quantified the radial displacement (decentration) response of a low-cost CCD camera with lens-retainer system and surrounding housing in shock and random environments [4]. Hatheway discusses a similar model applied to a forward looking infrared (FLIR) device [12]. Several groups have adopted a numerical approach to random vibration in idealized structures. An early contribution was made by Perkins who created a BASIC algorithm that returned the acceleration, velocity and displacement response of an SDOF structure [18]. Germane to this research is the work of Huang et al. who performed a closed-form analysis of a liquid crystal display (LCD) subjected to random white noise input to the base [13]. In this case, the display was idealized as a thin plate with transverse displacement and viscous damping. Ghanbari and Dunne measured the large-amplitude response of a clamped-clamped beam to random white noise and used the results to calibrate a SDOF forced random vibration model with a unique three-term non-linear damping model [10]. Moreover, they demonstrated the stationary Fokker-Plank (FPK) equation in conjunction with a finite element model to estimate 


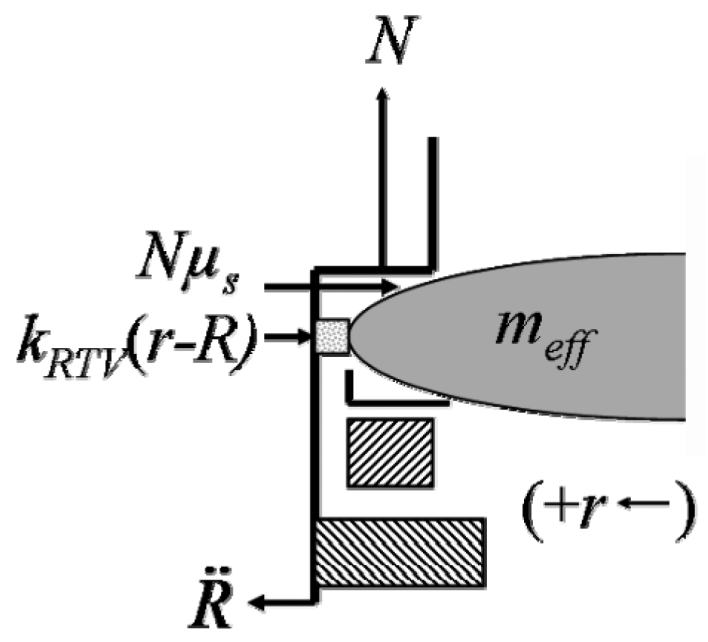

Fig. 2. (SDOF) mass-spring system.

extreme value (peak response) statistics. To the authors' knowledge, no previous work addresses peak response statistics for random vibration in a common lens-retainer-housing optomechanical system with Coulomb damping.

The present research includes a series of random vibration tests of optomechanical prototypes. Many groups have executed similar tests to quantify the impact of random vibration on the output of prototype electro-optical instruments. Comolli and Saggin executed a three-axis test of a space-based Fourier-transform infrared spectrometer [7]. Results led to a method of analyzing instrument spectrum phase information as a means of quantifying dynamicallyinduced signal corruption. Environmental tests of a pointing calibration and reference sensor (PCRS) by Mainzer et al. yielded characterizations of image position error (jitter) due to thermal excursions and random vibration [16]. Chen measured the response of an optical precision space flight apparatus to varying ground transportation inputs and obtained the dynamic parameters of the instrument [6]. Interferometry is widely used to indirectly determine alignment errors resulting from test. A recent paper by Irie et al. is one example [14]. Interferometry and related tools complement direct measurements of strain and displacement of individual optics. Sokolova discusses the so-called obliquity meter (contact profilometer) and the autocollimation telescope [21]. Recent work by Olson details a specialized microscopy apparatus for measuring manufacturing-induced decentration errors in precision injection-molded aspheres [17]. These are the basis for the unique coordinate measuring machine (CMM) method for measuring lens position and orientation presented here.

\section{Analysis}

\subsection{SDOF lens-retainer sub-assembly model}

Consider the lens - retainer sub-assembly of Fig. 1 (b) represented by the single-degree-of-freedom (SDOF) mass-spring system shown in Fig. 2. The free body diagram depicts the lens (and associated lens holder or edge support, not shown) with effective mass $m_{e f f}$ and input random acceleration $\ddot{R}$ applied to the housing. The RTV pad acts as a linear spring with stiffness $k_{R T V}$ and provides a restoring force proportional to the relative displacement $z$ between the lens and the housing denoted by the quantity $(r-R)$ in Fig. 2 [20]. A Coulomb or dry friction damping force is the product of the retainer pre-load $N$ and the coefficient of static friction $\mu_{s}$ between the lens and the housing. This force is assumed constant, acts in the plane parallel to the lens mount, and opposes lateral motion of the lens (note the gravity vector is assumed to be arbitrarily oriented orthogonal to the plane of the lens mount surface, into the page). To express the orientation of the damping force which depends on the sense of the lens relative velocity vector, the signum function is invoked, where: 


$$
\operatorname{sgn}(\dot{z})=\left\{\begin{array}{l}
+1(0<\dot{z}<+\infty) \\
0(\dot{z}=0) \\
-1(0>\dot{z}>-\infty)
\end{array}\right\} .
$$

Assuming the system is excited from rest and there is no hysteretic damping in the RTV spring, the equilibrium for the lateral motion of the lens relative to the housing is expressed as:

$$
m_{e f f}(\ddot{z}+\ddot{R})=-\operatorname{sgn}(\dot{z}) \mu_{s} N-k_{R T V} z .
$$

It follows that dividing though by the effective mass and re-arranging terms yields the equation of motion for the system:

$$
\ddot{z}+\operatorname{sgn}(\dot{z}) \frac{\mu_{s} N}{m_{e f f}}+\omega_{n}^{2} z=-\ddot{R}
$$

where $\omega_{n}$ is the natural frequency.

Input parameters including the coefficient of static friction, retainer pre-load, and RTV stiffness are estimates based on empirical data. The static friction coefficient is specific to the lens and housing materials and the retainer pre-load varies with the deflection of the wave spring (see Fig. 1). Specific parameters are presented with the model output in a later segment. The stiffness of the RTV pad may be approximated from the axial deflection model of a linearly-elastic, homogeneous, prismatic bar:

$$
k_{R T V}=\frac{A E}{L}
$$

where $A$ is the cross-sectional area, and $L$ is the pad length [9]. The RTV pad in this study is typically $5 \mathrm{~mm}$ in diameter (corresponding area of $\left.19.6 \times 10^{-6} \mathrm{~m}^{2}\right)$, and $0.254 \mathrm{~mm}(0.010$ inches) long. Product-specific references suggest a value of $4.83 \mathrm{MPa}(700 \mathrm{psi})$ for the modulus of elasticity, $E[1,2,8]$. It follows that the estimated RTV stiffness from Eq. (4) is $3.7 \times 10^{5} \mathrm{~N} / \mathrm{m}$. In a typical optomechanical installation, the lens is potted with multiple parallel RTV pads about the circumference.

\subsection{Peak response in stationary random vibration}

The issue of peak response of structures in stationary random vibration is important to understanding system reliability when a structure may fail due to passage of a measure of its response beyond a failure limit. There are closed form approximations to the peak response probability distribution of a simple, linear structure in stationary response, or the closely related first passage probability problem. Further, there are approximations to the peak response probability distributions of some simple, nonlinear structures, but these are rather limited. Here, we utilize a numerical approach for approximation of the peak response probability distribution of the nonlinear SDOF optomechanical assembly with equation of motion given in Eq. (3), subjected to wideband random excitation. The nonlinearity enters the problem through the Coulomb damping described above.

We assume that the SDOF system is excited through the housing with a zero-mean, stationary random excitation $\{\ddot{R}(t),-\infty<t<\infty\}$ in the time domain $(t)$. See, for example Wirsching, Paez and Ortiz, for the definition of a random process [22]. The excitation has Gaussian distribution, and its spectral density is $G_{\ddot{R} \ddot{R}}(f), 0<f<\infty$, where $f$ is the excitation frequency. The response random process can be approximated via Monte Carlo simulation. A summary of the Monte Carlo simulation steps follows. First, generate a discrete-time, finite duration realization $\ddot{r}_{j m}, j=0, \ldots, n-1$, of the excitation random process, $\{\ddot{R}(t),-\infty<t<\infty\}$ where the subscript $m$ indexes the realization and $n$ is the number of evaluation points. Next, compute the structural response $z_{j m}, j=0, \ldots, n-1$ from Eq. (3) to the generated excitation. This can be accomplished using any of a number of numerical techniques, such as the Runge-Kutta method [19]. Repeating the response calculation $M$ times generates an array of unique random excitations $\ddot{r}_{j m}, j=0, \ldots, n-1, m=1, \ldots, M$, and corresponding responses, $z_{j m}, j=0, \ldots, n-1, m=$ $1, \ldots, M$. Statistical analysis of the array yields an estimate of the probabilistic characteristics of the response random process.

A random process realization is generated via the formula 


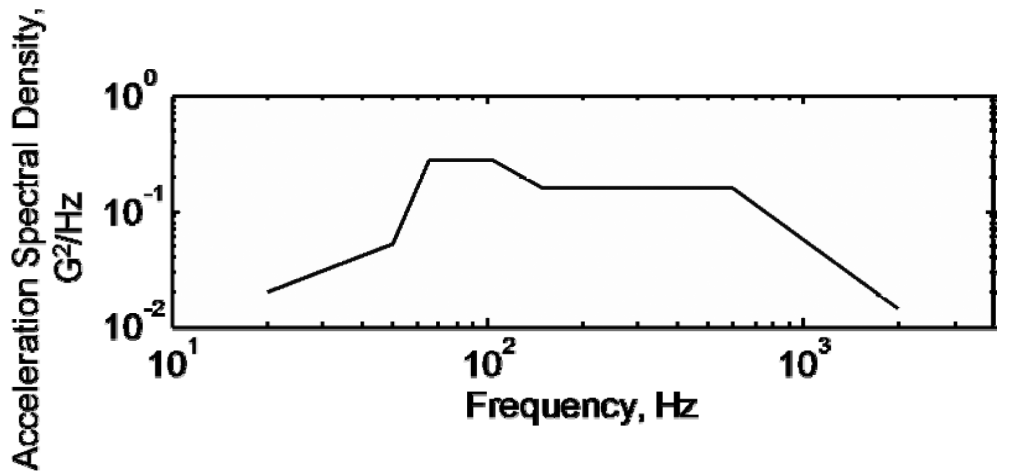

Fig. 3. Spectral density of a stationary random process.

$$
\ddot{r}_{j}=\frac{1}{n} \sum_{k=0}^{n-1} \eta_{k} e^{i 2 \pi j k / n} \quad j=0, \ldots, n-1
$$

where $\eta_{k}, k=0, \ldots, n-1$, is the discrete Fourier transform (DFT) of a discrete time, random signal realization. It may be recognized that the formula represents the inverse DFT of the quantity $\eta_{k}, k=0, \ldots, n-1$. The first half is defined as:

$$
\eta_{k}=\sqrt{\frac{n}{2 \Delta t} G_{\ddot{R} \ddot{R}}\left(f_{k}\right)} e^{i \phi_{k}} \quad k=0, \ldots, n / 2
$$

where $\Delta t$ is the time increment at which the random signals are generated, $G_{\ddot{R} \ddot{R}}\left(f_{k}\right), k=0, \ldots, n / 2$, is the spectral density of the random process $\{\ddot{R}(t),-\infty<t<\infty\}$ evaluated at the discrete frequencies $f_{k}=k \Delta f=$ $k / n \Delta t, k=0, \ldots, n / 2$, and $\phi_{k}, k=0, \ldots n / 2$, is a sequence of samples drawn from statistically independent, uniform random variables with range of realizations $(0,2 \pi)$. Equation (5) uses $\eta_{k}, k=0, \ldots, n-1$, for indices up to $n-1$, but Eq. (6) defines the DFT up to index $n / 2$, only. To obtain the additional values of $\eta_{k}$, we use:

$$
\eta_{n / 2+k}=\eta_{(n / 2)-k}^{*} \quad k=1, \ldots,(n / 2)-1 .
$$

In other words, the DFT of the random signal is Hermetian about the frequency index, $n / 2$. Figure 3 depicts the spectral density of a wide-band stationary random process. The corresponding finite duration realization generated using this method is shown in Fig. 4.

To characterize the response random process, we establish the probability distribution of random peak responses:

$$
Z_{\max , T}=\max _{0 \leqslant t \leqslant T}|z(t)| .
$$

That is, we seek the probability distribution of the maximum of the absolute value of the SDOF system displacement response within the time interval $[0, T]$. There are many means for obtaining $Z_{\max , T}$, and the one we choose to follow involves the framework provided by the Type I Asymptotic form for the distribution of extremes [5,11]. The Type I Asymptotic form provides the cumulative distribution function (CDF) for the largest value in a sequence of samples. The form of the $\mathrm{CDF}$ is:

$$
F_{Z_{\max }}(z)=\exp \left[-e^{-\alpha(z-u)}\right] \quad-\infty<z<\infty
$$

where the quantities $\alpha$ and $u$ are parameters of the distribution. The former quantity is called the scale parameter, and the latter quantity is called the location parameter. It is interesting to note that the mean and variance of a random variable who's CDF has the Type I asymptotic form are:

$$
\begin{aligned}
& \mu_{Z_{\max }}=u+\frac{\gamma}{\alpha}, \\
& \sigma_{Z_{\max }}^{2}=\frac{\pi^{2}}{6 \alpha^{2}},
\end{aligned}
$$




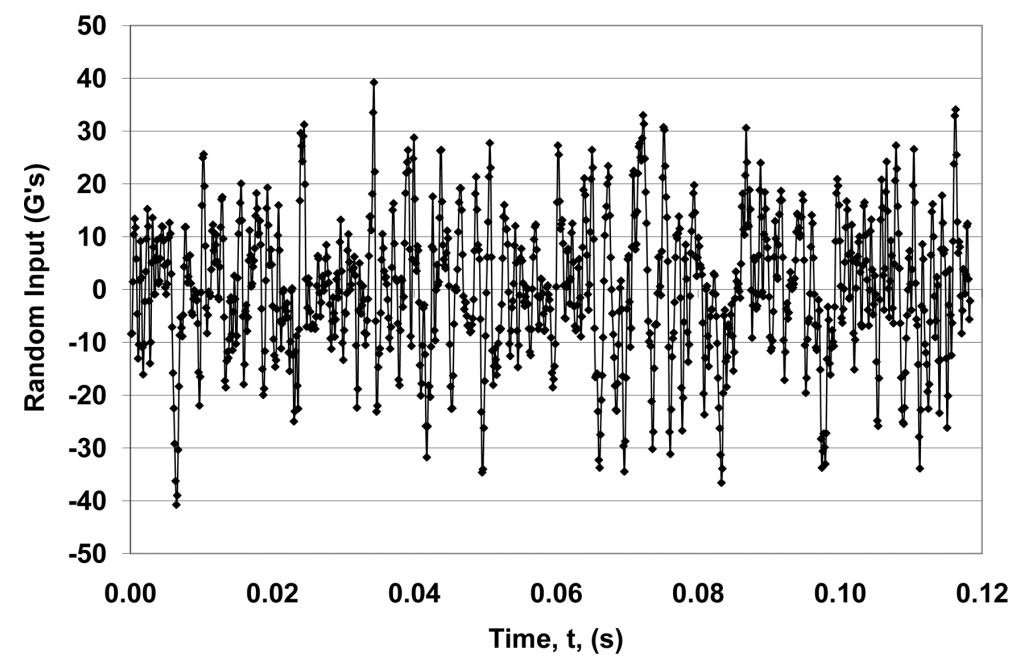

Fig. 4. Finite duration realization of a stationary random process.

respectively, where the constant $\gamma$, Euler's number, is defined $\gamma=0.577216 \ldots$ In this particular case, we take the parameters to be functions of the time interval duration, $T$, such that $\alpha$ is equal to $\alpha(T)$, and $u$ is equal to $u(T)$. In elementary settings involving structural dynamic response, it is sometimes possible to approximate the parameters, $\alpha$ and $u$, in terms of excitation parameters and structural dynamic system parameters, but that is more difficult for applications involving nonlinear and complex system responses. We can, however, use statistical means for estimating peak response probability distribution parameters. A method for peak response estimation follows.

Specify a sequence of time interval durations, $T_{k}, k=1, \ldots, N$, for which the parameters of Eq. (9) will be estimated. The values of $T_{k}$ should increase monotonically, and the largest of the values must satisfy $T_{N} \leqslant$ $n \Delta t$. For $k=1, \ldots, N$, we take each computed realization, $z_{j m}, j=0, \ldots, n-1, m=1, \ldots, M$, of the displacement response random process $\{Z(t),-\infty<t<\infty\}$, and separate it into non-overlapping segments of duration $T_{k}$. When $n \Delta t$ is not perfectly divisible into equal temporal segments $T_{k}$, define $N_{k}$ as the integer for which $\Psi_{k}=n \Delta t-N_{k} T_{k}$, belongs to the interval $\left[\Delta t, T_{k}-\Delta t\right]$. The first segment starts at a random time in that interval. Next, compute the realization of the random variable in Eq. (8), $z_{\max , T_{k}}$, within each signal segment. The result is a sequence of computed response peaks associated with the interval durations, $T_{k}, k=1, \ldots, N$. Write these as $\left(z_{\max , T_{k}}\right)_{i}, k=1, \ldots, N, i=1, \ldots, M N_{k}$. Each collection of peak data $\left(z_{\max , T_{k}}\right)_{i}, k=1, \ldots, N, i=1, \ldots, M N_{k}$, can be used to compute an empirical CDF. The empirical CDF of the kth set of data, $\left(z_{\max , T_{k}}\right)_{i}, i=1, \ldots, M N_{k}$, is created by sorting the data in ascending order; denote a collection of sorted peak values $y_{i}, i=1, \ldots, M N_{k}$. Use the $y_{i}, i=1, \ldots, M N_{k}$, as abscissa values in a plot where the ordinate is $\frac{i}{\left(M N_{k}\right)}, \mathrm{i}=1, \ldots, M N_{k}-1$, for abscissa values in the interval $\left[y_{i}, y_{i+1}\right]$. The empirical CDF equals zero for abscissa values less than $y_{1}$, and it equals one for abscissa values greater than or equal to $y_{M N_{k}}$. The result is a stair-step plot. Following the transformation, the CDF of Eq. (9) is then fit to the empirical CDF by a least squares approach, and this amounts to estimating the parameters $\alpha\left(T_{k}\right)$ and $u\left(T_{k}\right)$. Finally, the parameters $\alpha\left(T_{k}\right)$ and are plotted as functions of the discrete $T_{k}, k=1, \ldots, N$, and approximated using simple functional forms. The results can be used to establish the $\alpha\left(T_{k}\right)$ and $u\left(T_{k}\right)$ at interpolated (and, perhaps, slightly extrapolated) values of $T$ for the computation of probabilities that the random variable, $Z_{\max , T}$, will fall within or outside specified intervals.

\section{Experiment}

Random vibration tests were conducted in which the final position and orientation of sub-assembly optics (relative to a pre-test reference) was measured and compared to RMS and peak response statistics. Details of the test procedure and apparatus follow. 
Table 1

Prototype parameters for sub-assembly random vibration test

\begin{tabular}{lccccc}
\hline Prototype & $\begin{array}{c}\text { Housing } \\
\text { bore }(\mathrm{m})\end{array}$ & $\begin{array}{c}\text { Number of parallel } \\
\text { RTV pads }\end{array}$ & $\begin{array}{c}\text { Lens effective mass, } \\
m_{\text {eff }},(\mathrm{kg})\end{array}$ & $\begin{array}{c}\text { Interface coefficient of static } \\
\text { friction, } \mu_{s}\end{array}$ & $\begin{array}{c}\text { Retainer pre-load, } \\
N,(\mathrm{~N})\end{array}$ \\
\hline$H$ & 0.075 & 8 & 0.133 & 0.31 & 127 \\
$J$ & 0.057 & 8 & 0.105 & 0.21 & 114 \\
$M$ & 0.086 & 10 & 0.176 & 0.21 & 287 \\
\hline
\end{tabular}

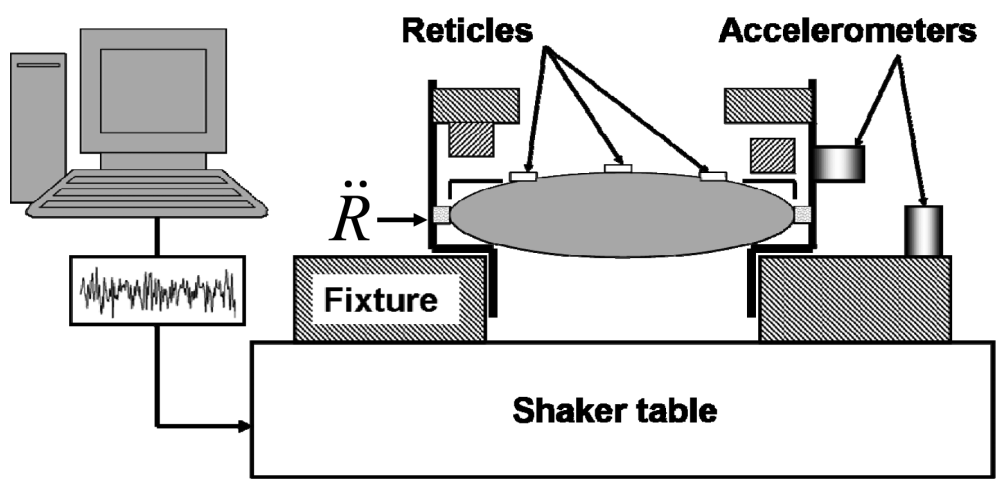

Fig. 5. Test apparatus schematic.

\subsection{Sub-assembly random vibration test}

Three prototype sub-assemblies of the configuration depicted in Fig. 1 were exposed to random vibration environments (see Fig. 3) input to the housing in the radial (lateral) direction for 180 seconds. Table 1 lists parameters, including housing dimensions, mass, coefficients of static friction, and retainer pre-load for prototype sub-assemblies designated $H, J$, and $M$. Pre-load is estimated during prototype assembly with a priori knowledge of the pitch of the mating threads in the housing that accept the retainer and the stiffness of the wave spring. Pre-load is the product of the reciprocal thread pitch, the number of turns of the threaded retainer during installation, and the wave spring stiffness. The wave spring compression is typically verified by measuring the final position of the retainer relative to the housing datum using a depth micrometer. Autocollimator and/or non-contact (video) CMM techniques were employed to gage the initial and final position and orientation of the lens relative to the housing. These are described in the next section. Double-pass transmission interferograms were generated for Prototype $M$ indicating image aberration content and optical alignment pre- and post test. Figure 5 is a schematic of the test apparatus configured for video CMM measurements. Following initial characterization, the instrumented prototype was mounted to a shaker table that delivered the random vibration environment. Data from accelerometers attached to the housing and table verified the lenses received random vibration with the intended spectral density.

\subsection{Lens position measurements}

The apparatus for measuring lens position and orientation of Prototypes $H$ and $J$ consists of a video CMM that images reticles attached to the lens surface. The reticles (approximately $1.5 \mathrm{~mm}$ square) are laser cut from 75-micrometer Kapton ${ }^{\circledR}$ tape with intersecting laser-scribed lines, approximately 25 micrometers wide, centrally located. In a typical vibration test, three reticles are arranged at equal intervals across the diameter. Given the subassembly dimensions listed in Table 1, the video CMM can measure the reticle positions relative to the housing with less than two micrometers uncertainty. A repeatability study was performed in which Prototype $H$ was removed from the CMM and re-registered. Precision of five micrometers was demonstrated. An overall response measurement process uncertainty is the sum of the accuracy and precision. When converted from English units and rounded up for conservatism, the process uncertainty is 7.8 micrometers. Comparing pre- and post test CMM measurements from two or more reticles leads to a data set that indicates final position and orientation. 


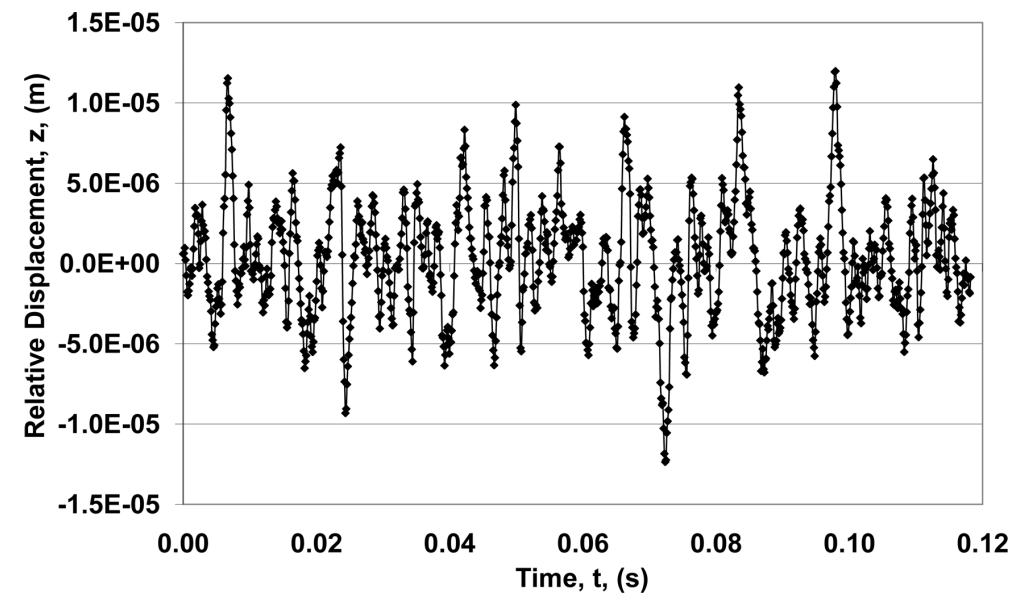

Fig. 6. Displacement response in the time domain, Prototype $M$.

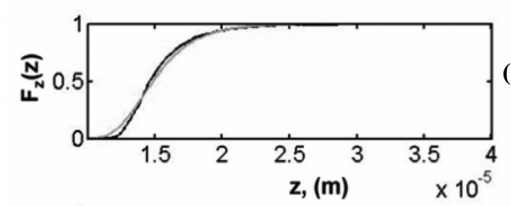

(a)
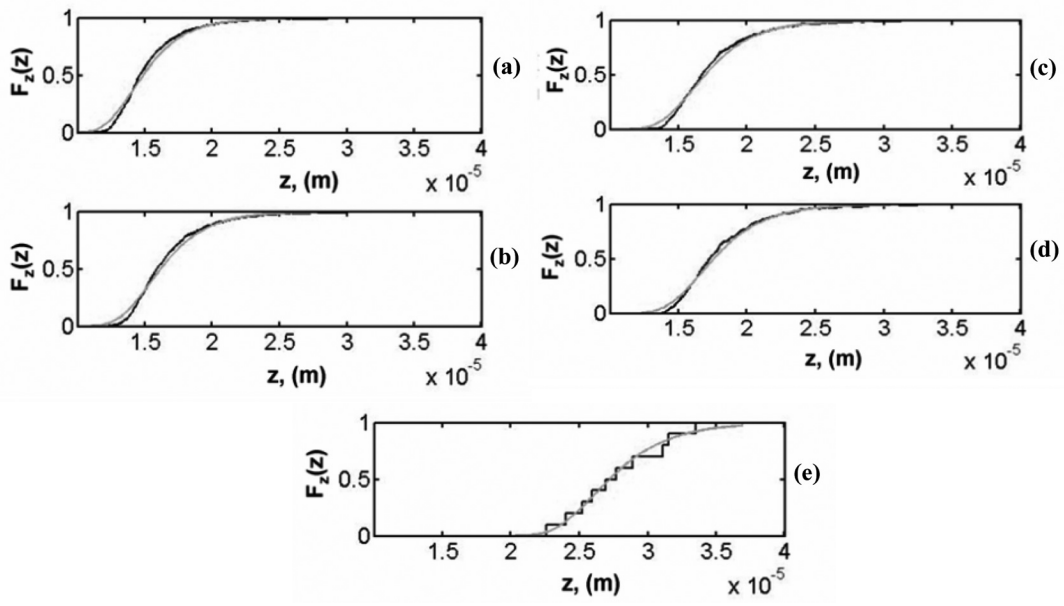

Fig. 7. Empirical CDFs of peaks for Prototype $M$ that occur in intervals of length (a) $T_{1}=1.2$ seconds, (b) $T_{2}=2.4$ seconds, (c) $T_{3}=3.6$ seconds, (d) $T_{4}=4.8$ seconds, and (e) $T_{150}=180$ seconds (dark stair-step curves) plotted with the CDF of the Type I asymptotic distribution of extremes (light curve).

Prototype $M$ is measured in terms of lens centration. Prior to test, the prototype sub-assembly is mounted to a commercial autocollimator coupled with an air bearing. Rotating the prototype on the air bearing under autocollimator interrogation yields a measure of lens centration relative to the optical (housing) axis with uncertainty comparable to the video CMM.

\section{Results}

Numerical results of root-mean-square (RMS) response and the peak response probability distribution analysis for the prototypes are reported in this segment. These are compared to experimental data.

\subsection{Peak response statistics}

The following sample calculation of peak response statistics considers Prototype $M$. Peak response statistics for all prototypes are summarized in the following section. A total of ten excitations similar to Fig. 4 were generated. 


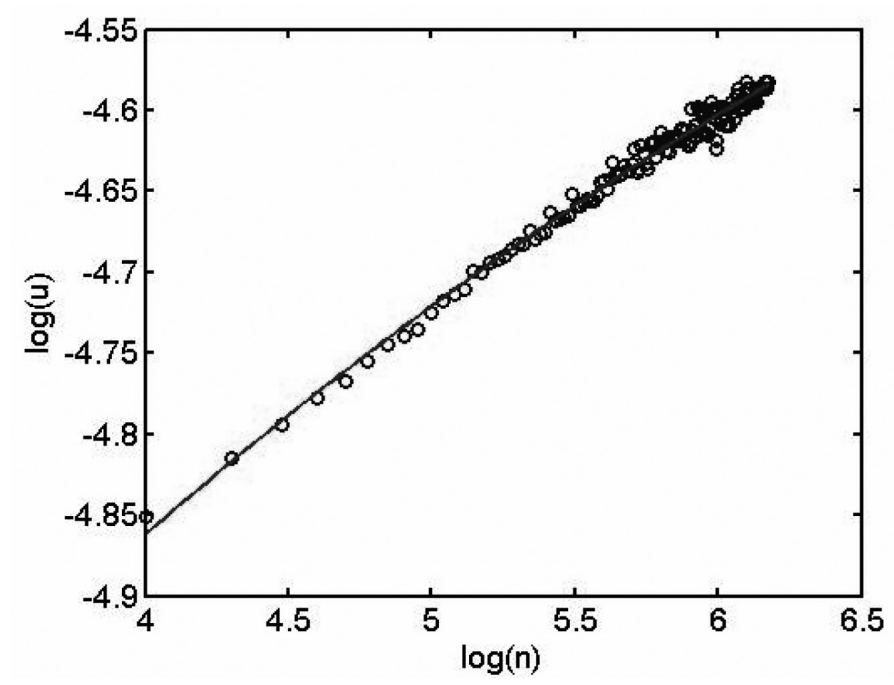

Fig. 8. Common logarithms of fitted location parameters as a function of common logarithms of the normalized time intervals.

The computation time increment $\Delta \mathrm{t}$ is $120 \mu \mathrm{s}$. Each signal contains $n=1.5 \times 10^{6}$ points. Therefore, the duration of each excitation is 180 seconds. The response to each excitation governed by Eq. (3), with parameters listed in Table 1, was computed using the Runge-Kutta approach. Figure 6 is a plot of displacement response in the time domain. Peak values from Eq. (8) were defined for time intervals of duration $T_{k}=1.2 \mathrm{k}$ seconds, where $k=1, \ldots$, 150. In the ten signals, there are 1500 intervals of duration $T_{1}=1.2$ seconds, 750 intervals of duration $T_{2}=2.4$ seconds, and ultimately ten intervals of duration $T_{150}=180$ seconds. Each interval contains one peak value. The empirical CDFs of peaks that occur in intervals of length $T_{1}=1.2$ seconds, $T_{2}=2.4$ seconds, $T_{3}=3.6$ seconds, $T_{4}=4.8$ seconds, $T_{150}=180$ seconds, are shown as the (darker curve) stair-step curves in Figs 7 (a), 7 (b), 7 (c), 7 (d), and 7 (e). The least squares fits to the empirical CDFs with the theoretical form of Eq. (9) (the CDF of the Type I asymptotic distribution of extremes) are plotted with the stair-step curves. The figures make it clear that the theoretical form fits the empirical CDFs nearly perfectly where many data are available, and very well when few data are available, indicating the aptness of the assumption of the theoretical form. Further, the shapes of the CDFs are nearly the same, indicating that the scale parameter $\alpha(T)$ is approximately constant for all the distributions. The CDFs shift to the right with increasing interval duration, and this indicates that the location parameter $u(T)$ is a function of the interval duration $T$. The reason for the right-shift of the CDF curves (Figs 7(a) through 7(e)) with increasing interval duration, $T$, is that higher peaks tend to be observed as the observation duration is increased.

The average scale parameter for all $150 \mathrm{CDF}$ fits is $\hat{\alpha}=3.284 \times 10^{5} \mathrm{~m}^{-1}$. The common logarithms of the fitted location parameters $u(T)$ of the CDFs are plotted as a function of common logarithms of the normalized time interval lengths $T_{k} / \Delta t$ in Fig. 8. Data points are depicted as circles. It is clear that $u(T)$ increases with interval length, $T$. We chose to model the variation in the logarithm of $u(T)$ as a quadratic in the logarithm of the normalized time interval length. The data were fit by a least squares method and the result is the estimated location parameter expressed as follows:

$$
\hat{u}(T)=\left(2.203 \times 10^{-6}\right)\left(\frac{T}{\Delta t}\right)^{0.2447-\left(11.507 \times 10^{-3}\right) \log (T / \Delta t)} \quad 0<T \leqslant 180 .
$$

The fitted curve is plotted in Fig. 8.

When the parameter estimates $\hat{\alpha}$ and $\hat{u}(T)$ are evaluated for a particular interval length $T$, and then used in Eq. (9), the CDF of peak values associated with the interval length is obtained. For example, when $T$ is equal to 180 seconds, the parameters are $\hat{\alpha}=3.284 \times 10^{5} \mathrm{~m}^{-1}$ and $\hat{u}(180)=2.602 \times 10^{-5} \mathrm{~m}$. The CDF of extreme values is:

$$
F_{Z_{\max }, 180}(z)=\exp \left[-e^{-\left(3.284 \times 10^{5}\right)\left(z-2.602 \times 10^{-5}\right)}\right] \quad-\infty<z<\infty
$$


Table 2

Peak response statistics and comparison to prototype experimental data

\begin{tabular}{|c|c|c|c|c|c|c|}
\hline Prototype & $\begin{array}{c}\text { Predicted average } \\
\text { RMS } \\
\text { response }(\mu \mathrm{m})\end{array}$ & $\begin{array}{c}\text { Average scale } \\
\text { parameter } \\
\left(\mathrm{m}^{-1}\right)^{*}\end{array}$ & $\begin{array}{c}\text { Estimated } \\
\text { location } \\
\text { parameter }(\mu \mathrm{m})\end{array}$ & $\begin{array}{c}\text { Probability of peak } \\
\text { response not exceeding } \\
\text { centration tolerance* }(\%)\end{array}$ & $\begin{array}{l}\text { Final position } \\
\text { (centration) from } \\
\text { experiment }(\mu \mathrm{m})\end{array}$ & $\begin{array}{c}\text { Measurement } \\
\text { uncertainty } \\
(\mu \mathrm{m})\end{array}$ \\
\hline$H$ & 3.31 & $2.08 \times 10^{5}$ & 31.0 & 3.03 & 2.5 & 7.8 \\
\hline$J$ & 2.35 & $2.57 \times 10^{5}$ & 31.4 & 83.33 & 5.1 & 7.8 \\
\hline$M$ & 3.63 & $3.28 \times 10^{5}$ & 26.0 & 0 & 2.5 & 4.0 \\
\hline
\end{tabular}

${ }^{*}$ Centration tolerances: $17 \mu \mathrm{m}$, Prototype $M ; 38 \mu \mathrm{m}$, Prototype $J ; 25 \mu \mathrm{m}$, Prototype $H$.

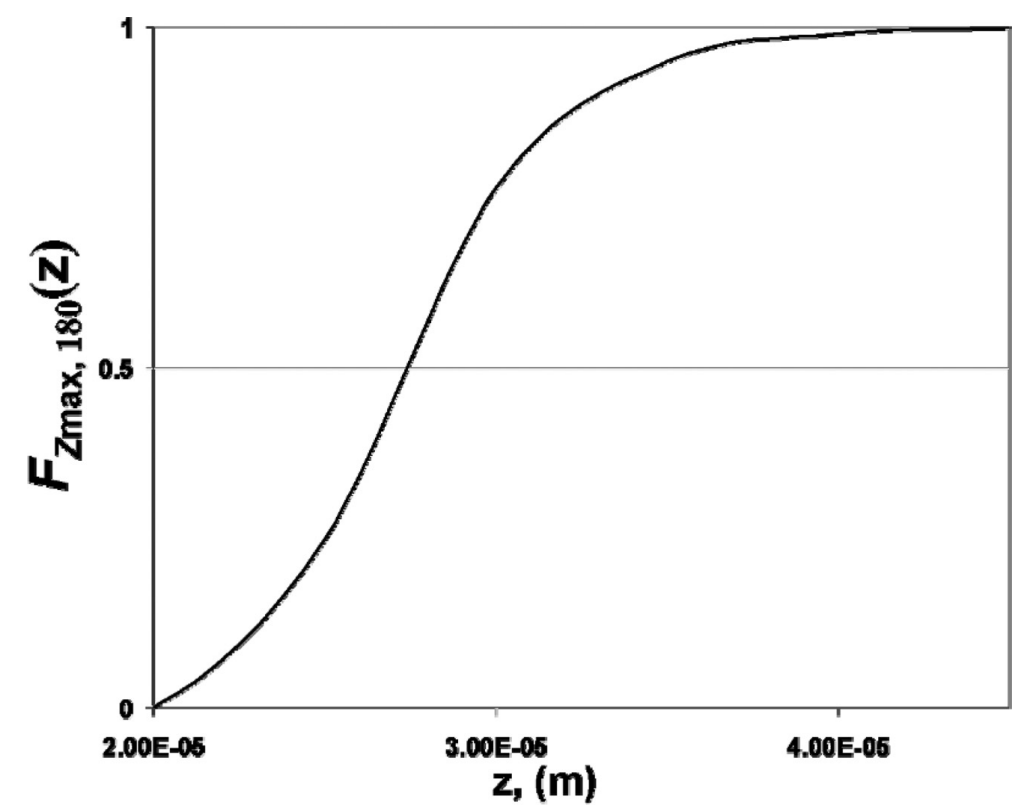

Fig. 9. CDF of extreme values corresponding to the response of Prototype $M$.

and is plotted in Fig. 9. The mean and standard deviation of the greatest peak response in an interval of length $T=$ 180 seconds is:

$$
\mu_{Z_{\max , 180}}=2.778 \times 10^{-5} \quad \sigma_{Z_{\max }, 180}=3.905 \times 10^{-6}(\mathrm{~m})
$$

It follows that the probability of a peak response in an interval of length $T=180$ seconds not exceeding the gap between the lens and the housing bore (250 micrometers) is 100\%. Likewise, the probability of the peak response not exceeding an arbitrary centration tolerance of 50 micrometers is $99.96 \%$.

\subsection{Comparison to experimental results}

Table 2 is a summary of analysis data and peak response statistics with experimental results. Test data in the table indicates that the maximum post-test centration is approximately five micrometers incurred by Prototype $J$. In each case, the centration is of comparable magnitude with the predicted average RMS response, suggesting that the prediction is reasonable. A comparison with design parameters listed in Table 1 reveals that average RMS response increases with lens effective mass. Centration is less than the measurement process uncertainty discussed above. It follows that in the experiment, the final centration for Prototype $H$ and $J$ can vary by the measured value plus or minus 7.8 micrometers. Actual lens centration tolerances are listed as a footnote to Table 2 and vary among the prototypes. The smallest tolerance ( 17 micrometers) is associated with Prototype $M$.Assigning parameter $z$ the tolerance value and evaluating Eq. (13) reveals that there is nearly zero probability that the peak response will not exceed the tolerance. Conversely, Prototype $J$ with the greatest centration tolerance (38 micrometers) exhibits a probability greater than $80 \%$ that peak response will not exceed the tolerance. A topical discussion of the results follows. 


\section{Discussion}

It is important to note the distinction between the RMS and peak responses which occur at one or more instances over the course of the random disturbance, and final centration that reflects the end state. The indirect comparison based on final centration data indicates the order of magnitude of the actual RMS and peak response and suggests that the analytical results are appropriate.

A factor that may contribute to bias in the analytical results is the inaccuracy in spectral density used for analysis relative to the spectral density of the random process that excites the physical system. Another is RTV stiffness. In practice, the spectral density bounds the range of expected random inputs. This conservatism certainly leads to over-predictions of the response. The dimensions of the RTV pads and corresponding high nominal stiffness precluded an experimental validation of the stiffness estimate. Under estimating the stiffness proportionally increases the magnitude of the response (see Eq. (3)). In the limiting case, the analysis may approximate the large unbounded response of a system in which the lens (with nominal pre-load, see Table 1) is not potted by assigning the stiffness parameter a value that approaches zero. In future work, empirical elastomer deflection models may improve the fidelity of stiffness estimates [15]. The significant impact of lateral stiffness on the system response is a key observation that has practical implications for optomechanical engineering design. Specifically, elastomer potting may be considered the primary means of constraining optics for random vibration environments. In the context of this work, the retainer assembly provides supplemental constraint that is not as effective. To illustrate, we calculate the average RMS response of Prototype $H$ (see Table 1) with pre-load increased to $132 \mathrm{~N}$, corresponding to the "full-down" configuration where the retainer assembly is advanced to the farthest point the housing design will allow, and the corresponding wave spring deflection is greatest. In the full-down configuration, we find that the average RMS response reported in Table 2 is unchanged. However, if the full-down response calculation is repeated with a low arbitrary radial stiffness (radial stiffness equal to unity, for example), the average RMS response increases by two orders of magnitude. Further quantitative investigations of this argument may require refinement of the Coulomb damping model and is reserved for future work.

\section{Conclusions and future work}

A numerical analysis was applied to predict the lateral response of optics in an optomechanical sub-assembly subject to random excitation of the housing. Prototype sub-assemblies underwent random vibration testing. Predictions were compared to experimental results with reasonable agreement. The Type I Asymptotic form for distribution of extremes was used to provide the cumulative distribution function (CDF) for peak response probabilities. Peak response statistics were calculated based on prototype design parameters.

In addition to comments in the previous section, future work involves further development of the analysis for use as a design and optimization tool. For example, the numerical analysis may be combined with a structural and optical model of the lens to calculate the retainer pre-load and lateral stiffness necessary for optimal balance of random vibration performance and lens strain (wave front error). Such optimizations may require expanding the numerical analysis to consider pre-load variations induced by the response of the lens along the optical axis. Finally, statistical analysis may be adapted to predict cumulative peak response probability distribution for stationary random vibration of assemblies of discrete optical elements.

\section{Acknowledgements}

The authors thank Pavel Chaplya, Zachary Kreiner, and James R. Wade of Sandia National Laboratories; and Brian Rider, Scott Svenson, and David Crompton of L-3 Communications SSG Corporation for their contributions to this research. Sandia National Laboratories is a multiprogram laboratory operated by Sandia Corporation, a Lockheed Martin Company, for the United States Department of Energy's National Nuclear Security Administration under contract DE-AC04-94AL8500. 


\section{References}

[1] 2009, NuSil CV-2566 Product Profile [Portable Document Format]. Available: http://www.nusil.com/library/products/CV-2566P.pdf.

[2] 2009, RTV566-Silicone Rubber Compound for Low Outgassing Applications. Available: http://www.gesilicones.com/siliconesweb/am1/ en/documents/datasheets/1469.html.

[3] A. Ahmad, Optomechanical Engineering Handbook. CRC Press,Boca Raton, FL, 1999.

[4] A. Ahmad et al., Optomechanical design of a low-cost CCD-based camera, Proceedings of the SPIE-The International Society for Optical Engineering 4093 (2000), 127-131.

[5] A.H.-S. Ang, Probability Concepts in Engineering Planning and Design, Volume II, Decision, Risk, and Reliability 2, John Wiley and Sons, New York, 1984.

[6] D. Chen, Research on environment vibration experiment upon optical precision spaceflight apparatus during transportation, Chinese Journal of Scientific Instrument 27 (2006), 711-714.

[7] L. Comolli and B. Saggin, Evaluation of the sensitivity to mechanical vibrations of an IR Fourier spectrometer, Review of Scientific Instruments 76 (2005), 123112-123111-123112-123112-123118.

[8] P. A.K.a.K. Dokania, Rliability Study of RTV 566 for Its Application as a Spring, IEEE Transactions on Device and Materials Reliability 8 (2008), 229-233.

[9] J.M. Gere and S.P. Timoshenko, Mechanics of Materials, 4 ed. PWS Publishing Company,Boston, 1997.

[10] M. Ghanbari and J.F. Dunne, An experimentally verified non-linear damping model for large amplitude random vibration of a clampedclamped beam, Journal of Sound and Vibration 215 (1998), 343-379.

[11] E.J. Gumbel, Statistics of Extremes. Columbia University Press, New York, 1958.

[12] A.E. Hatheway, Random image motion analysis with Ivory 2.0 FE models, Proceedings of the SPIE-The International Society for Optical Engineering $\mathbf{6 6 7 5}$ (2007), 66750E-66751-66750E-66750E-66710.

[13] W. Huang et al., A simplified random vibration analysis on portable electronic products, Components and Packaging Technologies, IEEE Transactions on 23 (2000), 505-515.

[14] S. Irie et al., Development for the alignment procedure of three-aspherical mirror optics, Proceedings of the SPIE - The International Society for Optical Engineering 3997 (2000), 807-813.

[15] P.B. Lindley, Engineering Design With Natural Rubber, in: NR Technical Bulliten, The Malaysian Rubber Producers' Research Association, Hertford, U.K., 1978.

[16] A.K. Mainzer et al., Pre-launch performance testing of the Pointing Calibration and Reference Sensor for SIRTF, Proceedings of the SPIE The International Society for Optical Engineering 4850 (2003), 122-129.

[17] C. Olson, Precision centration measurements on injection-molded lenses for digital imaging, Proceedings of the SPIE - The International Society for Optical Engineering $\mathbf{5 8 7 2}$ (2005), 587206-587201-587206-587206-587211.

[18] J. Perkins, Program calculates and plots vibration spectra, Machine Design 59 (1987), 116-118.

[19] W.H. Press, S.A. Teukolsky, W.T. Vetterling and B.P. Flannery, Numerical Recipes in C, The Art of Scientific Computing, Cambridge University Press,Cambridge, U.K., 1997.

[20] S.S. Rao, Mechanical Vibrations, 3 ed. Addison-Wesley Publishing Co.,New York, 1995.

[21] N.S. Sokolova, Lens decentering and deformation testing during the assembly of objectives, Soviet Journal of Optical Technology 40 (1973), 499-501.

[22] P.H. Wirsching, T.L. Paez and K. Ortiz, Random Vibrations - Theory and Practice. John Wiley and Sons, New York, 1995.

[23] P.R. Yoder, Opto-Mechanical Systems Design, 3 ed. SPIE Press,Bellingham, WA, 2006. 

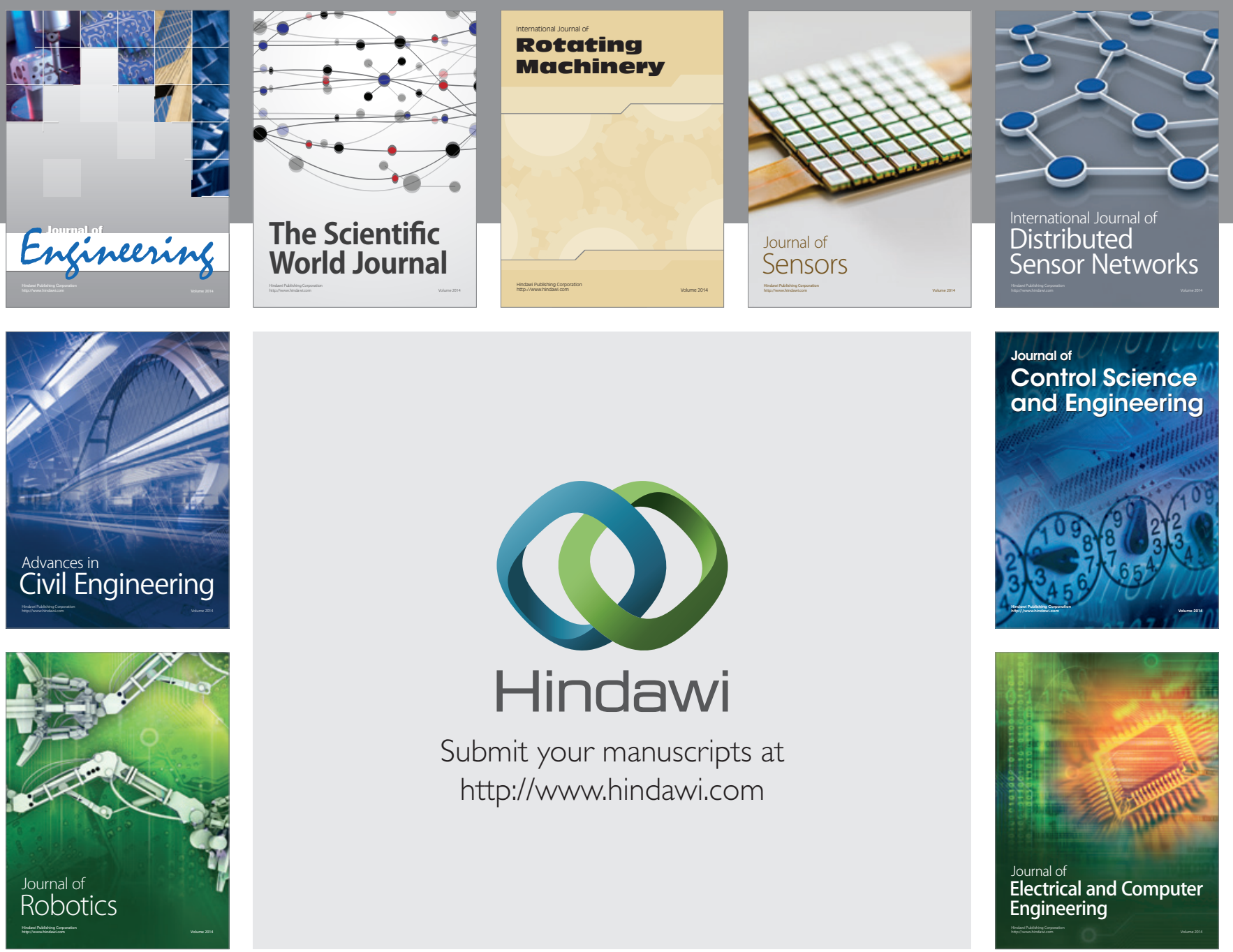

Submit your manuscripts at

http://www.hindawi.com
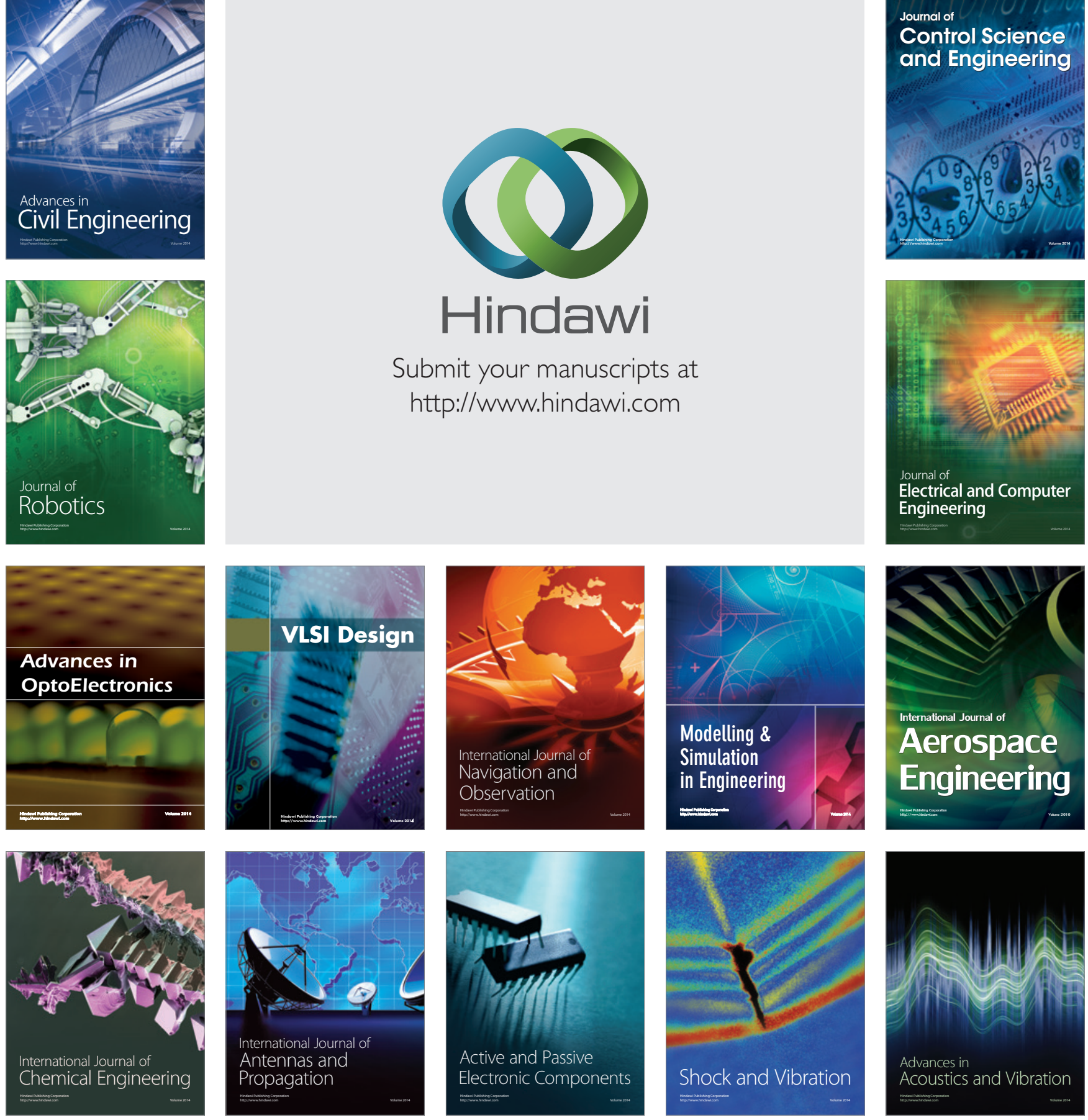